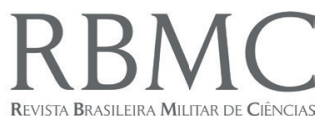

ISSN 2447-9071

doi https://doi.org/10.36414/rbmc.v7i18.98

Contato para correspondência: Roberpaulo Anacleto Neves

E-mail:

roberpaulo.neves@faculdadepm. edu.br

Conflito de interesse: Não

Financiamento: Recursos próprios

Recebido: 25/06/2021

Aprovado: 22/07/2021

\section{CAUSAS DA QUEDA PROGRESSIVA DAS TAXAS DE VACINAÇÃO PARA POLIOMIELITE NO BRASIL, NO ANO DE 2018}

\section{Causes of the progressive drop-in polio vaccination rates in Brazil in 2018}

\author{
Caroline Cunha Fernandes Carvalho', Frederico de Miranda Filho', Roberpaulo \\ Anacleto Neves ${ }^{1,2}$
}

'Pontifícia Universidade Católica de Goiás - PUC Goiás

${ }^{2}$ Faculdade da Polícia Militar - FPM

\section{Resumo}

O objetivo deste estudo foi realizar uma análise epidemiológica da cobertura vacinal para Poliomielite no Brasil em 2018. Trata-se de um estudo transversal com análise da cobertura vacinal de Poliomielite no Brasil, em 2018 e correlação com as variáveis: escolaridade, Índice de Desenvolvimento Humano (IDH), assistência à saúde e acesso à internet. A coleta de dados foi realizada a partir do Instituto Brasileiro de Geografia e Estatística (IBGE) e Programa Nacional de Imunização (PNI), DataSUS. Os limites espaciais obtidos por meio dos sites do Laboratório de Processamento de Imagens e Geoprocessamento (LAPIG) e Pastagem. Os dados e limites espaciais foram tabulados e processados pelo programa Microsoft Office Excel 2010 e gerados os mapas, em formato Shapefiles (SHP), com auxílio do software ArcGis. Nas regiões Norte e Nordeste as coberturas vacinais foram satisfatórias (acima de 95\%). Nas demais regiões as coberturas foram insatisfatórias na faixa etária entre um ano e dois anos incompletos. As regiões que não atingiram as coberturas adequadas foram as que apresentam maior IDH, maior escolaridade e maior acesso à internet, conforme esperado. A assistência à saúde não apresentou relação direta com os resultados. É necessária a elaboração de estratégias que objetivem alcançar as metas de vacinação em todo o país através da conscientização da população em relação a alguns temas como: a disseminação, principalmente pela internet, de conteúdos não confiáveis contra a vacinação; a importância de uma boa cobertura vacinal para a sociedade; o risco de epidemias de doenças que já haviam sido erradicadas; e a confiabilidade das vacinas.

Palavras-Chave: Cobertura vacinal; poliomielite; epidemiologia.

\section{Abstract}

The aim of this study was to carry out an epidemiological analysis of the vaccination coverage for Poliomyelitis in Brazil in 2018. This is a cross-sectional study analyzing the vaccination coverage of Poliomyelitis in Brazil in 2018 and correlation with the variables: education, Human Development Index ( $\mathrm{HDI}$ ), health care and internet access. Data collection was carried out from the Brazilian Institute of Geography and Statistics (IBGE) and the National Immunization Program (PNI), DataSUS. The spatial limits obtained through the sites of the Image Processing and Geoprocessing Laboratory (LAPIG) and Pasture. Data and spatial limits were tabulated and processed by the Microsoft Office Excel 2010 program and maps were generated, in Shapefiles (SHP) format, with the aid of ArcGis software. In the North and Northeast regions, vaccination coverage was satisfactory (above 95\%). In the other regions, coverage was unsatisfactory in the age group between one year and two incomplete years. The regions that did not reach adequate coverage were those with higher $\mathrm{HDI}$, higher education and greater access to the internet, as expected. Health care was not directly related to the results. It is necessary to develop strategies that aim to achieve vaccination goals across the country through raising the population's awareness in relation to some topics such as: the dissemination, mainly on the internet, of unreliable content against vaccination; the importance of good vaccination coverage for society; the risk of disease epidemics that had already been eradicated; and the reliability of vaccines. Keywords: Vaccine coverage; polio; epidemiology. 


\section{Introdução}

A Poliomielite é uma doença infectocontagiosa que acomete indivíduos de qualquer faixa etária, com maior ocorrência, principalmente em crianças. Esta doença é causada por infecção pelo poliovírus, cujo período de incubação viral varia de dois a 30 dias. $\mathrm{O}$ aspecto clínico da Poliomielite é bastante amplo, que varia desde formas assintomáticas ou oligossintomáticas, semelhante a quadros virais (febre, dor de garganta), até a forma paralítica da doença, com sequelas permanentes, insuficiência respiratória e morte. Na forma paralítica ocorre perda da força muscular e reflexo sem perda da sensibilidade $\mathrm{e}^{1,2}$.

Nos primeiros anos de vida, os indivíduos acometidos passam por episódios de internações longas e inúmeros procedimentos médicos, portanto, além do dano físico, há um enorme dano psicossocial gerado pelo isolamento, separação da família e amigos, sentimentos de abandono, desamparo, medo, entre outros. Mesmo após a reabilitação, ainda existem muitas dificuldades a serem enfrentadas por sua condição física e pela barreira social aos deficientes físicos ${ }^{3-5}$.

Ao avaliar a gravidade das sequelas, tanto precoce, quanto tardias e o fato de não existir medicamento efetivo para o tratamento, torna-se necessário rever as estratégias de prevenção da doença. Deve-se considerar a obtenção de dados epidemiológicos para orientar as estratégias, uma vez que existem vacinas seguras e imunogênicas que podem assegurar a erradicação da doença ${ }^{6,7}$.

A campanha nacional de vacinação contra a Poliomielite é realizada pelo Ministério da Saúde do Brasil (MS) em parceria com as secretarias estaduais e municipais de saúde. Esta estratégia tem como objetivo manter elevada a cobertura vacinal contra a Poliomielite, com intuito de evitar a reintrodução do vírus selvagem da Poliomielite, para manter o estado de eliminação dessa doença no país. Estas campanhas foram iniciadas em 1980, sendo o país livre da doença após 1990. A população alvo desta ação é composta de crianças de um a cinco anos incompletos e a meta mínima a ser alcançada corresponde a 95\% de cobertura vacinal contra Poliomielite ${ }^{4,8,9}$.

Apesar dos progressos alcançados desde o início do programa global de erradicação da Poliomielite, a doença permanece endêmica em alguns países (Afeganistão, Nigéria e Paquistão) e outros são considerados de risco para o agravo (aqueles com baixa cobertura vacinal, bolsões de não vacinados e que mantêm viagens internacionais ou relações comerciais com estes países). Portanto, reforça-se a necessidade da realização da campanha de vacinação para Poliomielite, a fim de alcançar crianças ainda não vacinadas ou que não obtiveram resposta imunológica satisfatória, minimizando o risco de adoecimento dessas crianças e, consequentemente, reduzindo ou eliminando os bolsões de não vacinados. No Brasil, as coberturas vacinais municipais ainda são heterogêneas e isso possibilita a reintrodução do poliovírus selvagem (comprometendo o processo de certificação de eliminação). Desta forma, é importante que autoridades, gestores e profissionais de saúde trabalhem para minimizar os riscos e a possibilidade de reintrodução do poliovírus no território brasileiro. A disseminação dessas informações de saúde pode permitir a tomada de decisões baseadas em evidências e a programação das ações de saúde. Assim, o estudo objetiva realizar análise epidemiológica da cobertura vacinal de Poliomielite no Brasil em $2018^{4,8,10,11}$.

\section{Métodos}

Trata-se de um estudo transversal descritivo com análise da cobertura vacinal de Poliomielite no Brasil, referente ao ano de 2018 e correlação com as variáveis: índice de desenvolvimento humano municipal (IDHM), índice de desenvolvimento humano municipal educação (IDHM Educação), percentual de pessoas que utilizaram a internet na população de 10 anos ou mais de idade, proporção de usuários coberto por equipe de saúde da família (ESF). A coleta de dados foi realizada a partir da base de dados do Instituto Brasileiro de Geografia e Estatística (IBGE) e Programa Nacional de Imunização (PNI), DataSUS, os limites espaciais obtidos por meio dos sites do Laboratório de Processamento de Imagens e Geoprocessamento (LAPIG) e Pastagem. Os dados e limites espaciais foram tabulados e processados pelo programa Microsoft Office Excel 2010 e gerados os mapas, em formato Shapefiles (SHP), com auxílio do software ArcGis. Como o estudo foi realizado a partir de dados de domínio público informatizados, não foi necessária a apreciação do Comitê de Ética em Pesquisa (CEP), segundo a CONEP 466/12 $12-14$.

\section{Resultados}

Como pode ser observado na Figura 1, na sequência, nas regiões Norte e Nordeste as coberturas vacinais foram satisfatórias (acima de 95\%). Nas demais regiões as coberturas foram insatisfatórias na faixa etária entre um ano e dois anos incompletos, sendo que na região Sul a cobertura vacinal da referida faixa etária foi $89,22 \%$; na região Sudeste foi $86,9 \%$ e na região Centro Oeste foi $89,68 \%$.

Em relação ao índice de desenvolvimento humano (IDH) das regiões foram (Gráfico 1): norte - 0,667; nordeste - 0,663; sudeste - 0,766; sul -0,754 e centro-oeste -0,757. O índice de desenvolvimento em educação (IDHM) das regiões foram: norte - 0,557; nordeste - 0,569; sudeste -0,688; sul -0,662 e centro-oeste - 0,665, representando o nível de escolaridade. 


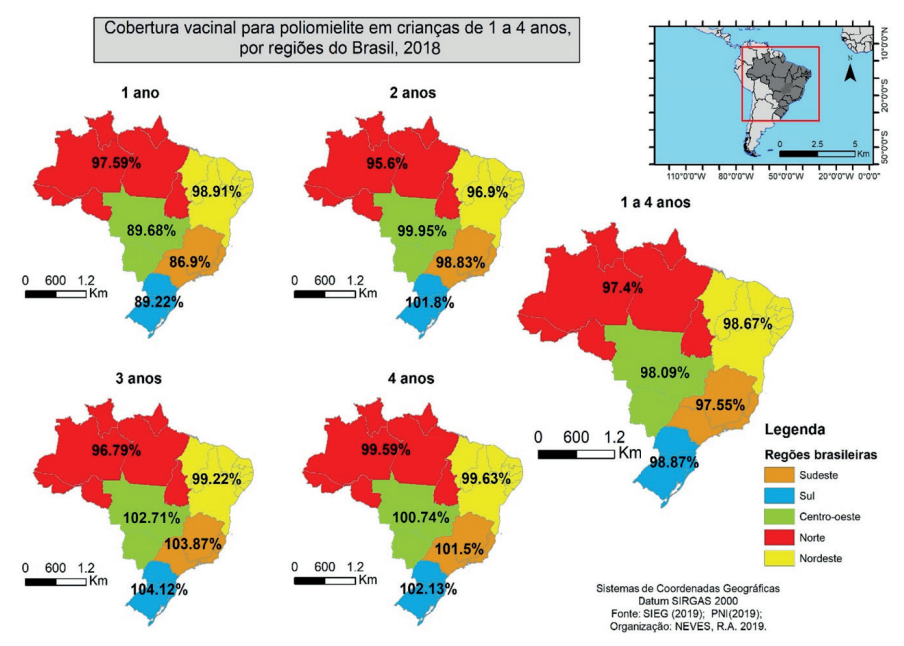

Figura 1. Cobertura vacinal para poliomielite em crianças de 1 a 4 anos, por regiões do Brasil, referentes ao ano de 2018.

Gráfico 1. Relação do IDHM e IDHM Educação dos estados brasileiros.

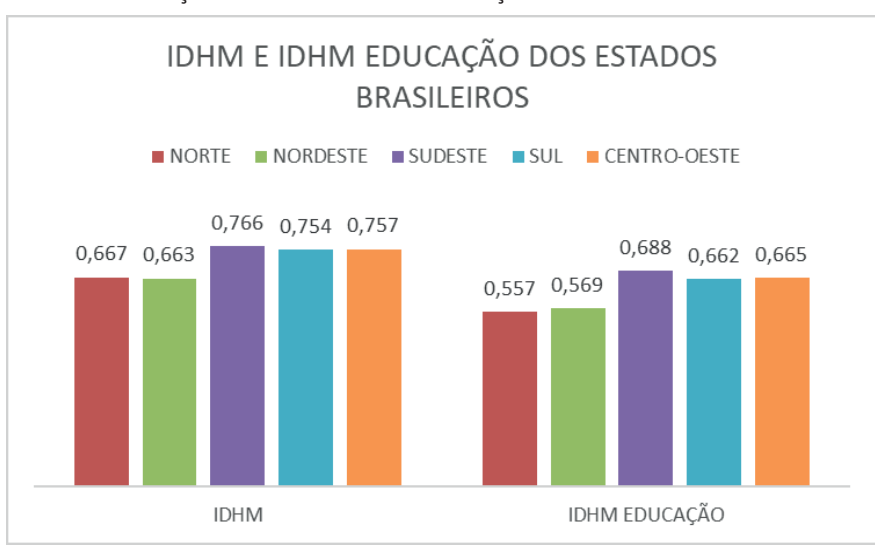

Fonte: Índice de desenvolvimento humano e educação nas macrorregiões brasileiras: 2018. - Brasília: PNUD: IPEA: FJP, 2018.

Quanto a utilização da internet via dispositivos móveis ou desktop/notebook/tablets, o percentual de pessoas que utilizaram a internet foi maior nas regiões que não atingiram a cobertura vacinal (Gráfico 2): norte $-46,2 \%$; nordeste $-45,1 \%$; sudeste $-65,1 \%$; sul $-61,1 \%$ e centro-oeste $-64 \%$.

Gráfico 2. Percentual de pessoas que utilizaram a internet na população de 10 anos ou mais de idade.

\section{PERCENTUAL DE PESSOAS QUE UTILIZARAM}

A INTERNET NA POPULAÇÃO DE 10 ANOS OU MAIS DE IDADE
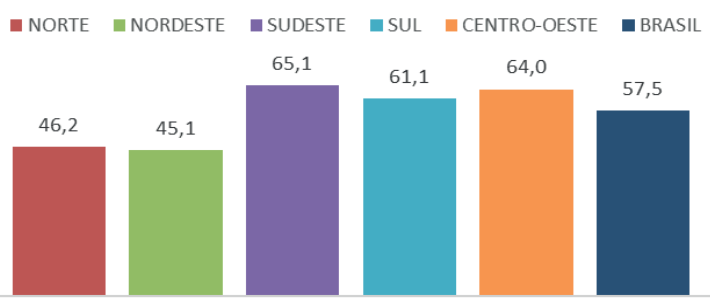

UTILIZARAM INTERNET

Fonte: IBGE, Diretoria de Pesquisas, Coordenação de Trabalho e Rendimento, Pesquisa Nacional por Amostra de Domicílios 2018.
Já a proporção de usuários cobertos por equipe ESF, que representa a assistência à saúde, não apresentou relação direta com os resultados (Gráfico 3): norte - 5183,9; nordeste $-3673,2$; sudeste $-6121,5$; sul $-4842,4$ e centro-oeste $-5343,6$.

Gráfico 3. Proporção de usuários cobertos por equipes de saúde da família.

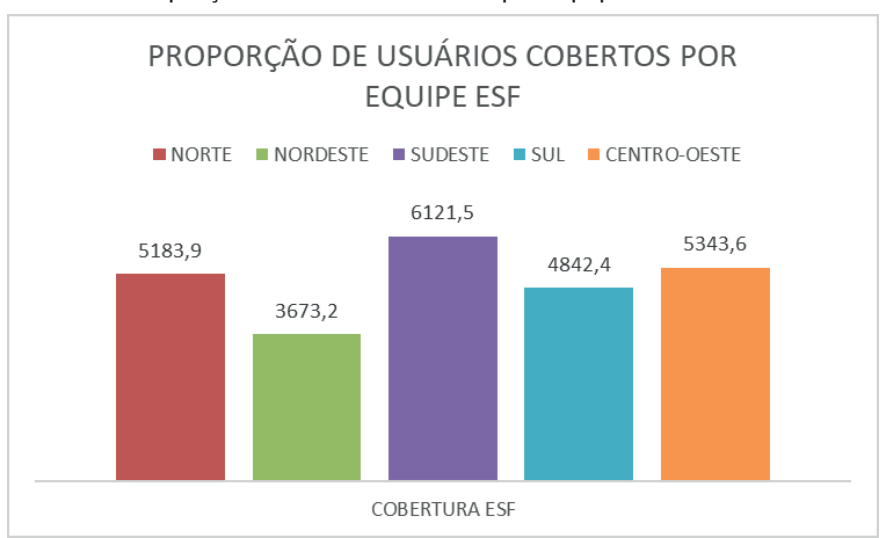

Fonte: IBGE 2018 e DataSUS, 2018.

\section{Discussão}

Em levantamento realizado sobre o monitoramento da cobertura vacinal para Poliomielite, entre 1990 e 2016, foi satisfatório, ou seja, acima de $95 \%$ da população alvo, a partir de então, se iniciou um declínio na adesão da população persistindo até os dias atuais ${ }^{15}$.

Quanto ao acesso à internet, a população que apresentou melhor nível socioeconômico, maior acesso à internet e maior IDH, exibiu menor cobertura vacinal nas regiões, chegando a $86,9 \%$ em crianças com até dois anos incompletos, enquanto nas regiões com menor IDH e menor índice de desenvolvimento em educação e menor acesso aos recursos digitais, a cobertura foi satisfatória para todas as faixas etárias. Neste contexto, estudos comparando diferentes países demonstraram que a alta escolaridade e status socioeconômico favoráveis não aumentam a taxa de vacinação. Os países que apresentaram as menores taxas de vacinação foram os que apresentam os níveis mais altos de escolaridade e bom acesso aos serviços de saúde ${ }^{15}$.

Podemos citar prováveis razões para a ocorrência da queda da vacinação: percepção enganosa de que esta não é necessária, uma vez que a doença desapareceu; desconhecimento do Programa Nacional de Imunização (PNI); temor de reações adversas ou sobrecarga do sistema imunológico; falta de tempo dos cuidadores. Além disso, há profissionais de saúde que desaconselham determinadas vacinas. Outra razão citada é a falta de confiança por parte da população, em relação à eficácia e formulação das vacinas ${ }^{15-17}$.

A cobertura vacinal também é influenciada pelo vínculo entre as famílias e os serviços de saúde, sendo maior nas 
unidades públicas, onde as crianças têm maior probabilidade de estar com o cartão vacinal atualizado. Nestes serviços os profissionais são mais treinados para incentivar a vacinação. Além desses fatores, a queda da cobertura vacinal também se dá por movimentos antivacinas, há relatos da ocorrência dos mesmos no ano de 1800 contra a vacina da varíola, quando grande parte da população a percebeu como invasão de sua liberdade. No Brasil a manifestação mais conhecida foi a Revolta da Vacina em 1904 15,16,18.

Podemos perceber que os movimentos antivacinas estão crescendo e sendo fortalecidos graças ao aumento de informações incorretas compartilhadas pelas mídias digitais. Parte da população é influenciada por notícias falsas que circulam nas redes sociais e ações de grupos contrários à imunização. $\mathrm{Na}$ Europa e nos Estados Unidos, são comuns grupos que se embasam em notícias inverídicas ou alegam razões religiosas e filosóficas ${ }^{15,16,19}$.

A queda da cobertura vacinal pode trazer grandes consequências, como epidemias frequentes de doenças que são imunopreveníveis (a exemplo do que está ocorrendo com o sarampo), além do risco de uma reintrodução do vírus selvagem da Poliomielite em nosso país ${ }^{15}$.

\section{Conclusão}

Conclui-se que as coberturas vacinais não foram satisfatórias nas regiões Sul, Sudeste e Centro-Oeste do país, locais onde foram observados melhores dados sociodemográficos e culturais - melhor IDHM, maior acesso à informação e melhores índices educacionais.

Portanto é necessário a elaboração de estratégias que objetivem alcançar as metas de vacinação em todo o país através da conscientização da população em relação a alguns temas como: a disseminação, principalmente pela internet, de conteúdos não confiáveis contra a vacinação; a importância de uma boa cobertura vacinal para a sociedade; o risco de epidemias de doenças que já haviam sido erradicadas; e a confiabilidade das vacinas.

\section{Referências}

1. Brasil. Conselho Nacional de Secretários de Saúde: CONASS. Coleção Progestores - Para entender a gestão do SUS [Internet]. 2007. 278 p. Available from: http:// bvsms.saude.gov.br/bvs/publicacoes/colec_progestores_livro6a.pdf

2. Hull HF, Ward NA, Hull BP, Milstien JB, de Quadros C. Paralytic poliomyelitis: seasoned strategies, disappearing disease. Lancet 1994;343:1331-7.

3. Brasil. Ministério da Saúde. Secretaria de Vigilância em Saúde. Departamento de Vigilância das Doenças Transmissíveis B. Biblioteca Virtual em Saúde do Ministério da Saúde www.saude.gov.br/bvs [Internet]. 2014. Available from: http://editora.saude.gov.br

4. Brasil. Ministerio da Saúde. Secretaria de Vigilancia em Saúde. Departamento de Vigilancia Epidemiologica. Acidentes por Animais Peçonhnetos. In: Guia de vigilância em saúde. Ministério. Brasilia: Ministério da Saúde. Ms. 2014;1:719-38.

5. Brasil. Diretrizes de Atenção à Reabilitação da Pessoa com Síndrome pòs-Poliomielite e Co-morbidades / Ministério da Saúde, Diretrizes de Atenção á Reabilitação com Síndrome Pós- Poliomelite e Co-comorbidades / Ministério da Saúde, Secretaria de Atenção à Saúde. 2016.

6. Bricks LF, Moraes JC de. Riscos da poliomielite para adolescentes e adultos na era da pré-erradicação global. Rev Pan-Amazônica Saúde. 2014;4(2):63-72.

7. Statement M. Post Polio Syndrome Management and Treatment in Primary Care. Building. 2007.

8. Edição N, Silva A, Atlanta CDC, Branco CMC, Fm F. Boletim Epidemiológico Paulista 117. 2013;14:3-24. Available from: http://www.saude.sp.gov.br/resources/ccd/ homepage/bepa/edicoes-2013/edicao_117_-_outubro_especial_carrapatos_parte_1.pdf

9. Eduardo MB de P. Vigilância Epidemiológica das Paralisias Flácidas Agudas/Manutenção da Erradicação da Poliomielite - importância, atividades, rotinas, fluxos operacionais, avaliação e pactuação. 2007; Available from: ftp://ftp.cve.saude.sp.gov.br/doc_tec/hidrica/ doc/if_pfapolio.pdf

10. Informe técnico campanha nacional de vacinação contra a poliomielite e o sarampo Santa Catarina. 2018.

11. Brasil. Ministério da Saúde. Manual dos Centros de Referência para Imunobiológicos e speciais. 2006;188.

12. IBGE. Censo 2010: Síntese das das Etapas da da Pesquisa. 2010;27.

13. Sistema estadual de geoinformação - SIEG 2017. Disponível em: http://www2.sieg.go.gov.br/post/ ver/169642/geoprocessamento Acesso em: 07 mar. 2019.

14. Instituto nacional de pesquisas espaciais - INEP. Introdução à Ciência da Geoinformação. Ministério da Ciência e Tecnologia. NPE-10506-RPQ/249. São José dos Campos, 2001.

15. Sato APS. Qual a importância da hesitação vacinal na queda das coberturas vacinais no Brasil ? Rev Saude Publica. 2018;1-9. 
16. Zorzetto R. As razões da queda na vacinação. Pesqui Fapesp 2018; (270):19-24.

17. Sousa CDJ, Vigo ZDL, Palmeira CS. Compreensão Dos Pais Acerca Da Importância Da Vacinação Infantil. Rev Enferm Contemp. 2015;1(1):44-58.

18. Vasconcellos-Silva PR, Castiel LD, Griep RH. A sociedade de risco midiatizada, o movimento antivacinação e o risco do autismo. Cien Saude Colet [Internet]. 2015;20(2):607-16. Available from: http://www.scielo.br/scielo.php?script=sci_ arttext\&pid=S1413-81232015000200607\&lng=pt\&t lng=pt

19. Shimizu NR. Revista do Edicc. Rev do EDICC - ISSN 2317-3815 [Internet]. 2018;5(5):87-97. Available from: http://revistas.iel.unicamp.br/index.php/edicc/article/ view/5963/7310. 